Revue musicale OICRM

revue musicale oicrm

\title{
Claude Debussy vu par Jean Cocteau
}

\section{Malou Haine}

Volume 2, numéro 1, 2014

La réception de Debussy au XXe siècle. Incidences, influences et autorité

URI : https://id.erudit.org/iderudit/1055852ar

DOI : https://doi.org/10.7202/1055852ar

Aller au sommaire du numéro

\section{Éditeur(s)}

OICRM

\section{ISSN}

2368-7061 (numérique)

Découvrir la revue

Citer cet article

Haine, M. (2014). Claude Debussy vu par Jean Cocteau. Revue musicale OICRM, 2(1), 191-211. https://doi.org/10.7202/1055852ar

\section{Résumé de l'article}

Dans Le Coq et l'Arlequin (1918), Cocteau est le porte-parole de Satie et des jeunes musiciens qui gravitent autour de lui. Une vingtaine d'aphorismes attaquent violemment la musique de Debussy, car Cocteau souhaite voir émerger une musique française débarrassée des caractéristiques de la musique impressionniste. Par la suite, ses écrits abordent encore Debussy à plusieurs reprises, dans une bonne vingtaine d'articles divers. Jusque vers 1940 , il justifie ces positions de jeunesse ; il tente de se dédommager en tentant d'expliquer que c'était là une attitude de jeunes en rébellion contre leurs aînés. Par la suite, Cocteau sera de plus en plus nuancé ; il ne se justifiera plus, mais affirmera son admiration pour Debussy et son chef-d'oeuvre, Pelléas et Mélisande, dont il conçoit même les décors et les costumes lors d'une production au Théâtre municipal de Metz en 1962. 


\title{
Claude Debussy vu par Jean Cocteau
}

\author{
Malou Haine
}

\begin{abstract}
Résumé
Dans Le Coq et l'Arlequin (1918), Cocteau est le porte-parole de Satie et des jeunes musiciens qui gravitent autour de lui. Une vingtaine d'aphorismes attaquent violemment la musique de Debussy, car Cocteau souhaite voir émerger une musique française débarrassée des caractéristiques de la musique impressionniste. Par la suite, ses écrits abordent encore Debussy à plusieurs reprises, dans une bonne vingtaine d'articles divers. Jusque vers 1940, il justifie ces positions de jeunesse ; il tente de se dédommager en tentant d'expliquer que c'était là une attitude de jeunes en rébellion contre leurs aînés. Par la suite, Cocteau sera de plus en plus nuancé ; il ne se justifiera plus, mais affirmera son admiration pour Debussy et son chef-d'œuvre, Pelléas et Mélisande, dont il conçoit même les décors et les costumes lors d'une production au Théâtre municipal de Metz en 1962.
\end{abstract}

Mots clés : Cocteau ; Debussy ; écrits ; France ; littérature.

\begin{abstract}
In his well-known Le Coq et l'Arlequin (1918), Cocteau is the leader of Satie and the other young musicians that fostered Satie's model. More than twenty aphorisms are directed brutally against Debussy's music, because Cocteau wishes to see a French music avoided of characteristics that belonged to impressionist music. In the aftermath, his writings evoked Debussy a couple of time, precisely in twenty varied articles. Until 1940, Cocteau justified the positions that he developed against Debussy when he was young: he tried to legitimate himself with the explanation that his position was an attitude of young rebellions against their elders. After that step, Cocteau will be more nuanced in front of Debussy; he will no more justified himself as long as he claimed his admiration for Debussy and his chef d'oeuvre, Pelléas et Mélisande - he conceived the decors and costumes for the production of the opera that took place in the Théatre municipal de Metz in 1962.
\end{abstract}

Keywords: Cocteau; Debussy; France; literature; writings. 
Dans ses nombreux écrits, Jean Cocteau n'a pas toujours eu la même opinion vis-à-vis de Claude Debussy entre 1911 et $1963^{1}$. Certains aphorismes du manifeste intitulé Le Coq et l'Arlequin (1918) sont manifestement des attaques contre Debussy et son esthétique. Mais les textes qui ont suivi sont nettement plus nuancés, allant même jusqu'à l'expression contraire, tant par souci de réparation que par souhait de participer à la gloire posthume et unanime du compositeur.

Avant Le CoQ et L'ARLEQUiN

Avant Le Coqetl'Arlequin, Cocteau n'avait aucune attitude hostile à l'égard de Debussy. Au contraire, il le fréquentait assidûment au début des années 1910, ainsi qu'il le confie lui-même au journaliste de Radio-Lausanne à Henri Jaton en 1962 (Héron 2010).

En 1911, Cocteau est alors très lié à Gabriele D'Annunzio et à Ida Rubinstein qui préparent ensemble Le Martyr de saint Sébastien sur une musique de Claude Debussy et des décors de Léon Bakst. Cocteau assiste à toutes les répétitions de ce mystère dansé tenant à la fois d'un oratorio, d'un mimodrame et d'un ballet, mis en scène par Armand Bour dans une chorégraphie de Michel Fokine. Ce spectacle ne fait pas partie des Ballets russes, car dès le mois d'août 1909, Ida Rubinstein désirait s'émanciper de l'autorité de Diaghilev pour lequel elle avait incarné Cléopâtre en 1909 et Schéhérazade l'année suivante. Issue d'une famille russe fortunée, elle finance elle-même Le Martyr de saint Sébastien qui est créé au Théâtre du Châtelet, le 21 mai 1911, dans le cadre de la " Grande Saison de Paris » de Gabriel Astruc.

Dix jours après la création de ce ballet, Cocteau (1911) fait paraître un compte rendu élogieux sur l'interprète principal dans son rôle multiple à la fois dansé, parlé et chanté. Pas un mot, pas une phrase ne sont consacrés à Debussy, pas plus d'ailleurs qu'aux autres collaborateurs de l'œuvre, pas même à D'Annunzio, tant Cocteau est subjugué par Ida Rubinstein.

Il en va de même deux ans plus tard lorsque Cocteau consacre un nouvel article à ce spectacle dans un ouvrage d'hommages à Léon Bakst. Cette fois encore, le poète met l'accent uniquement sur Ida Rubinstein, ce qui semble normal, car à l'époque, Cocteau s'intéresse uniquement aux ballets. Il apparaît en quelque sorte comme un "critique chorégraphique ", avant de mettre sa plume au service de la critique musicale dans les années 1920.

Cocteau agit de même dans son texte consacré à une répétition du ballet Prélude à l'après-midi d'un faune ${ }^{2}$ : une seule allusion à la musique cependant, avec l'évocation de la flûte (qui représente le faune, dansé par Nijinsky) juste avant la fin du spectacle : "Couple, adieu. La Syrinx mineure termine son thème. Le rideau tombe " (1912), phrase poétique qui illustre la réexposition du thème à la tierce mineure. Mais aussi phrase descriptive qui n'implique aucune appréciation de la musique.

1 Pour l'ensemble des quelque trois cents textes de Cocteau sur la musique, voir Gullentops et Haine (en préparation). Ne sont cependant pas repris dans la présente étude les allusions à Debussy dans sa correspondance ou dans son Journal.

$2 \quad$ Prélude de l'après-midi d'un faune est créé par les Ballets russes le 28 mai 1912 au Théâtre du Châtelet dans des décors et costumes de Léon Bakst. 
Dans l'ouvrage consacré à Bakst, Cocteau revient sur ce spectacle, en mettant en évidence le danseur et en précisant qu'il n'apprécie ni le décor ni les costumes. Mais cette fois encore : rien sur la musique de Debussy. Certes, il s'agit d'une partition créée en 1894 à la Société nationale, alors que pour Le Martyr, la musique avait été spécialement commandée au compositeur. En d'autres termes, lorsque Cocteau parle d'Ida Rubinstein dans Le Martyr, il oublie ou évite de mentionner le nom du compositeur de la musique. Ce n'est que dans un texte de 1960 que son nom est enfin associé aux autres membres de cette production : "Et plus tard [après Cléopâtre], elle devint le saint Sébastien de Gabriele D'Annunzio, de Claude Debussy et de Bakst ».

En 1913, Cocteau assiste aux répétitions et à la création (29 mai 1913) du Sacre du printemps et rencontre souvent Debussy. En 1962, il se rappelle - mais est-ce un souvenir réel ou un souvenir inventé comme en a coutume notre poète? - que Debussy avait la partition sur les genoux et qu'il exprimait une " sorte de tristesse de voir tout à coup un nouveau monde musical » (Héron 2010) se présenter après le sien. Cocteau insiste : il ne s'agit pas d'une jalousie, mais d'une " sorte de mélancolie ».

Plus d'autre texte sur Debussy avant le fameux Coq et l'Arlequin. Pourtant Cocteau aurait pu se prononcer sur le ballet Jeux, monté par Diaghilev sur une musique de Debussy et créé le 15 mai 1913 au Théâtre des Champs-Élysées, éclipsé il est vrai par le succès-scandale du Sacre quelques jours plus tard. Cocteau évoquera bien plus tard ce ballet en écrivant un texte d'hommage à Diaghilev dans l'Encyclopédie du théâtre contemporain en 1957. Il se contente de dénoncer la chorégraphie médiocre de Vaslav Nijinski, mais ne dit rien de la musique :

Dans une assez médiocre chorégraphie de Jeux, Serge de Diaghilev et Claude Debussy se serraient l'un contre l'autre dans l'ombre de la salle, de fort mauvaise humeur, à cause d'une stylisation décorative que Nijinski prétendait opposer aux charmes impressionnistes $([1957])^{3}$.

\section{Le CoQ et L'ARLEQUin - Notes AUTOUR DE LA MUSIQue}

Lorsqu'on examine cet ouvrage, il convient d'être particulièrement attentif à la version utilisée, car il en existe quatre différentes, présentant des variantes significatives pour certaines d'entre elles.

La date de la première édition est généralement retenue comme 1918 ; or l'ouvrage paraît en réalité en janvier 1919. Il fait l'objet d'une "nouvelle édition " en 1918, en réalité septembre 1919, et diffère de la version précédente par la suppression d'un seul aphorisme. L'ouvrage est également réédité dans Le rappel à l'ordre (1926) avec, cette fois, de nombreuses suppressions et corrections. Mais on dispose aussi d'une série d'épreuves datées, pour l'envoi, du 27 juillet 1918 et, pour la réception chez l'éditeur, du 5 septembre 1918.

3 Toutes les citations de Cocteau seront ici référenciées par leur date de publication ou de rédaction placée entre crochets. Pour la bibliographie complète de tous les textes de Cocteau sur la musique, voir Gullentops et Haine (en préparation). 
Sans aucun doute, au vu des différences encore existantes avec la première édition, Cocteau avait reçu une seconde série d'épreuves. Remarquons qu'au moment où paraît la première édition, en janvier 1919, Debussy est mort depuis bientôt un an et n'a donc pas pu prendre connaissance de cet ouvrage. Les discussions avec Satie ont-elles pu lui donner un avant-goût de ce qui se tramait contre lui ?

Dans Le Coq et l'Arlequin ${ }^{4}$, Jean Cocteau se veut avant tout le défenseur d'Erik Satie. Il rejette le flou de l'impressionnisme de Debussy et les influences germano-slaves. Dans ce recueil d'aphorismes, Cocteau cite abondamment Satie et sa musique, mais il ne se trouve qu'une seule allusion - exprimée par antiphrase - à ce groupe en formation qu'est celui des Six :

*Ce livre ne parle d'aucune école existante, mais d'une école que rien ne fait pressentir, sinon les prémices de quelques jeunes, l'effort des peintres, et la fatigue de nos oreilles ${ }^{5}$ [Cet aphorisme signifie : les jeunes compositeurs ne doivent pas se laisser influencer par leurs aînés].

Cependant, c'est bien la musique des Six que Cocteau défend en insistant sur les caractéristiques souhaitées de cette musique française qui doit se colorer aux éléments de la vie quotidienne comme le cirque, le music-hall, le jazz ou le café-concert. On comprend tout de suite qu'il s'agit de la musique de Parade (Haine, à paraître) composée par Satie avec des éléments de bruits de la vie commune introduits à la demande de Cocteau, mais utilisés seulement lors de la reprise du ballet en 1920 (machine à écrire, aéroplane...).

Rappelons que ce ballet fut créé le 18 mai 1917 au Théâtre du Châtelet par les Ballets russes de Diaghilev sous la direction d'Ernest Ansermet. Satie est alors le chef de file des " Nouveaux Jeunes », bientôt baptisés « Le Groupe de Six ». La musique française prônée par Cocteau doit donc, par opposition, se débarrasser des influences étrangères, principalement celles de Richard Wagner, mais aussi celles de la musique russe qui a imprégné la musique de Debussy.

Sur un ensemble de quelque 215 aphorismes, 25 attaquent le compositeur de Pelléas. Cocteau procède d'abord par allusions :

*Un jeune homme ne doit pas acheter de valeurs sûres [Ce qui signifie : les jeunes compositeurs ne doivent pas se laisser influencer par leurs aînés].

*Tout « Vive Un Tel !» comporte un : «À bas Un Tel ! ». Il faut avoir le courage de cet « À bas Un Tel ! » sous peine d'éclectisme.

*L'éclectisme, c'est la mort de l'amour et de l'injustice. Or en art, la justice, c'est une certaine injustice [Ce qui signifie : défendre Satie et les jeunes, c'est renier Debussy, donc forcément être injuste envers celui-ci].

4 Sauf mention contraire, les aphorismes sont tirés de la première édition du Coq et l'Arlequin (1918).

5 Comme dans l'édition originale, chacun des aphorismes est précédé d'une étoile, ici remplacée par un astérisque. 
Les attaques envers « les musiciens impressionnistes » deviennent plus directes, et la marque du pluriel utilisée à l'encontre de ceux-ci ne trompe personne, car c'est bien Debussy seul qui est visé et frappé frontalement, parfois même très cruellement :

${ }^{*}$ Ce qui balaye la musique impressionniste c'est, par exemple, une certaine danse américaine que j'ai vue au Casino de Paris [Cet aphorisme se poursuit par une description du jazz band américain].

*[...] On se demande souvent pourquoi Satie affuble ses plus belles œuvres de titres drôles ${ }^{6}$ qui déroutent le public le moins hostile. Outre que ces titres protègent son œuvre des personnes en proie au « sublime » et autorisent à rire de ceux qui n'en ressentent pas la valeur, ils s'expliquent par l'abus debussyste des titres précieux. Sans doute faut-il voir là une mauvaise humeur de bonne humeur, une malice contre les Lunes descendant sur le temple qui fut, les Terrasses des audiences du Clair de lune et les Cathédrales englouties.

*Les musiciens impressionnistes coupèrent la poire en douze et donnèrent à chacun des douze morceaux un titre de poème. Alors, Satie composa douze poèmes et intitula le tout: Morceaux en forme de poire ${ }^{7}$.

*Satie a connu le dégoût de Wagner en pleine Wagnérie, au cœur même de la Rose-Croix. Il prévint Debussy contre Wagner. «Attention, lui disait-il, un arbre du décor ne se convulse pas parce qu'un personnage entre en scène ». C'est l'esthétique de Pelléas.

*Debussy a dévié, parce que de l'embûche allemande, il est tombé dans le piège russe. De nouveau, la pédale fond le rythme, crée une sorte de climat flou, propice aux oreilles myopes. Satie reste intact. Écoutez les Gymnopédies, d'une ligne et d'une mélancolie si nettes. Debussy les orchestre, les brouille, enveloppe d'un nuage l'architecture exquise. De plus en plus, Debussy s'écarte du point de départ posé par Satie et entraîne tout le monde à sa suite. La grosse brume trouée d'éclairs de Bayreuth devient le léger brouillard neigeux taché du soleil impressionniste. Satie parle d'Ingres ; Debussy transpose Clause Monet à la russe. Or, tandis que Debussy épanouissait délicatement sa grâce féminine, promenant Stéphane Mallarmé dans Le jardin de l'Infante, Satie continuait sa petite route classique. Il nous arrive aujourd'hui, jeune entre les jeunes, trouvant enfin sa place, après vingt ans de travail modeste.

*Quand je dis " le piège russe ", "l'influence russe ", je ne veux pas dire par là que je dédaigne la musique russe. La musique russe est admirable parce qu'elle est la musique russe. La musique française russe ou la musique française allemande est forcément bâtarde, même si elle s'inspire d'un

6 Sur les épreuves du texte, l'adjectif « drôles » est remplacé par celui de « bouffons ».

7 Les Trois Morceaux en forme de poire comprennent en réalité sept pièces et non douze (Orledge [1990]1990, p. 286-287). 
Moussorgski, d'un Stravinski, d'un Wagner, d'un Schoenberg. Je demande une musique française de France.

*La profonde originalité d'un Satie donne aux jeunes musiciens un enseignement qui n'implique pas l'abandon de leur originalité propre. Wagner, Stravinski et même Debussy, sont de belles pieuvres. Qui s'approche d'eux a du mal [pour 1918; à 1926] se dépêtrer de leurs tentacules; Satie montre une route blanche où chacun marque librement ses empreintes [Le terme pieuvre est utilisé pour signifier que ces compositeurs entraînent avec eux une série de suiveurs].

*Satie regarde peu les peintres et ne lit pas les poètes, mais il aime à vivre où la vie grouille; il a l'instinct de la bonne auberge; il profite d'une température.

*Debussy intronise le climat Debussy une fois pour toutes. Satie se transforme. Chaque œuvre intimement liée à l'œuvre précédente se détache pourtant d'elle et vit d'une vie propre. C'est une pâte originale, une surprise, - une déception pour ceux qui veulent qu'on piétine sur place.

*Assez de nuages, de vagues, d'aquariums, d'ondines et de parfums la nuit ; il nous faut une musique sur la terre, UNE MUSIQUE DE TOUS LES JOURS [Allusions directes aux titres de certaines œuvres de Debussy].

*Les musiciens impressionnistes ont cru que l'orchestre de Parade était pauvre parce qu'il était sans sauce.

*L'impressionnisme vient de tirer son joli feu d'artifice à la fin d'une longue fête. C'est à nous de bourrer les pétards d'une autre fête [Il est nécessaire de reconstruire quelque chose de neuf sur les ruines de l'impressionnisme].

*On ne blâme pas une époque, on se félicite de n'en avoir pas été.

*Mettre en garde contre une décadence n'est pas nier la valeur individuelle de ses artistes.

*L'impressionnisme est un contrecoup de Wagner. Les derniers roucoulements de l'orage.

*L'école impressionniste substitue le soleil à la lumière et la sonorité au rythme.

*Debussy a joué en français, mais il a mis la pédale russe [Le terme « pédale » revient très souvent sous la plume de Cocteau pour signifier une musique amplifiée par des artifices divers].

*Le jeu latin se joue sans mettre les pédales ; le romantisme enfonce les pédales. Pédale Wagner; Debussy pédale ${ }^{8}$. 
*Naturellement Wagner, c'est très bien, Debussy, c'est très bien ; on ne parle que des choses très bien. Il est inutile de dire que Saint-Saëns, Bruneau, Charpentier, c'est très mal ${ }^{9}$.

* «Autour d'un Picasso et d'un Braque, autour d'un Stravinski et d'un Satie, que de farceurs qui les discréditent ! » Ainsi juge l'impressionniste. Sans doute oublie-t-il le Salon d'automne, et le cheveu en quatre de Mélisande.

*Pelléas, c'est encore de la musique à écouter la figure dans les mains. Toute musique à écouter dans les mains est suspecte. Wagner, c'est le type de la musique qui s'écoute dans les mains.

* On ne peut pas se perdre dans le brouillard Debussy comme dans la brume Wagner, mais on y attrape du mal.

On constate bien que Cocteau pondère légèrement ses propos anti-debussystes lorsqu'il reconnaît qu'il y a une certaine injustice à renier les aînés et lorsqu'il affirme que « mettre en garde contre une décadence n'est pas renier la valeur individuelle de ses artistes ». Cette " pédale douce » apportée à ses propos, pour utiliser le propre langage du poète, restera insuffisante aux yeux de ses contemporains et surtout à ceux des générations suivantes qui ont considéré Le Coq et l'Arlequin comme une philippique à l'égard de Debussy.

Dès lors, Cocteau n'aura de cesse de se justifier dans ses écrits ultérieurs. Dans ce manifeste, il précisera avoir voulu préciser " certains périls et certaines promesses " (1921), d'où la nécessite d'utiliser des expressions grossissantes pour se faire mieux comprendre.

\section{ERIK SATIE, INITIATEUR DE L'ESTHÉTIQUe IMPRESSIONNISTE ?}

Une assertion souvent avancée par Cocteau dans ses écrits consiste à prétendre que Satie a ouvert la voie de l'impressionnisme à Debussy. À plusieurs reprises, il fait allusion à cet événement, mais il est plus explicite dans un article de 1924 dont nous reproduisons en entier les passages incriminés :

C'est en 1891 que Satie compose la musique d'une « wagnérie » de Péladan ${ }^{10}$ et ouvre sans que personne [ne] s'en doute la porte par laquelle Debussy va marcher vers la gloire.

Debussy fréquentait alors l'auberge du Clou, mal vu des artistes de gauche parce qu'il venait d'avoir le Prix de Rome. On l'évitait. Un soir, Debussy et

9 Cet aphorisme sera supprimé dans l'édition de 1926. C'est donc déjà un signe évident de la volonté de Cocteau d'atténuer sa pensée par rapport à 1918.

10 Le fils des étoiles, musique de scène pour flûtes et harpes pour la Pastorale Kaldéenne en trois actes de Joséphin Péladan, édité en 1891 à Beauvais, à l'Imprimerie professionnelle. Seule l'édition de $1920 \mathrm{chez}$ Rouart-Lerolle porte l'inscription « Wagnérie kaldéenne ». 
Satie se trouvent à la même table. Ils se plaisent. Satie demande à Debussy ce qu'il prépare. Debussy composait, comme tout le monde, une " wagnérie » avec Catulle Mendès ${ }^{11}$. Satie fit la grimace. "Croyez-moi, murmura-t-il, assez de Wagner. C'est beau, mais ce n'est pas de chez nous. Il faudrait... »

Ici, je vais citer une phrase de Satie qui m'a été dite par Debussy et qui décida l'esthétique de Pelléas et Mélisande.

« Il faudrait, dit-il... que l'orchestre ne grimace pas quand un personnage entre en scène. Regardez. Est-ce que les arbres du décor grimacent ? Il faudrait faire un décor musical, créer un climat musical où les personnages bougent et causent. Pas de couplets, pas de leitmotiv - se servir d'une certaine atmosphère de Puvis de Chavannes ».

Pensez à l'époque dont je parle. Puvis de Chavannes était un audacieux, moqué par la droite.

« Et vous, Satie, que préparez-vous »? demanda Debussy.

«Moi, dit Satie, je pense à La princesse Maleine ${ }^{12}$, mais je ne sais pas comment obtenir l'autorisation de Maeterlinck ».

Quelques jours après, Debussy, ayant obtenu l'autorisation de Maeterlinck, commençait Pelléas et Mélisande ${ }^{13}$.

Ne croyez pas que je vais blâmer Debussy, plaindre Satie. Tant mieux. Le chef-d'œuvre est à qui le décroche.

Un chef-d'œuvre n'ouvre rien, n'annonce rien. Il ferme une période. Point, à la ligne. Voilà le chef-d'œuvre. Il faut passer à la ligne. C'est dans le chef-d'œuvre que viennent se cristalliser mille recherches confuses, mille plasmas, mille ébauches, mille tâtonnements. Le coup de génie de Satie fut de comprendre tout de suite, dès 1896, que Pelléas était un chef-d'œuvre, de reconnaître généreusement et astucieusement que son ami Claude avait tiré dans le mille.

[...] Satie avait, sans le savoir, imaginé la musique impressionniste ${ }^{14}$ [1924].

Par le simple fait d'une suggestion, Satie serait donc à l'origine de l'esthétique de Pelléas! Cocteau n'hésite donc pas à détourner les propos rapportés pour en magnifier

11 Debussy est alors occupé à mettre en musique Rodrigue et Chimène, opéra en trois actes sur un livret de Catulle Mendès.

12 La princesse Maleine, première pièce de Maurice Maeterlinck datant de 1889.

13 Selon François Lesure (2003, 522), Debussy obtient, au mois d'août 1892, l'autorisation de Maeterlinck de mettre en musique Pelléas, quelques mois après avoir acheté le texte en librairie.

14 Si Cocteau reprend, comme le titre l'indique, des fragments d'un texte de 1920, il modifie cependant certains passages. Les passages cités ici datent pour la plupart de 1924 et non de 1920. 
la portée. Nous utilisons ici le terme « magnifier » à la manière de Cocteau lui-même, c'est-à-dire pour sa polysémie, ses deux significations, celle de grandir et d'idéaliser avec celle de glorifier et célébrer. Cocteau se trouve donc constamment sur le fil rouge de la vérité, si celui-ci peut servir sa démonstration.

Un chef-d'œuvre finit par être supplanté par un autre chef-d'œuvre. Stravinsky évincera Debussy :

Un beau jour, le chef-d'œuvre destiné à contredire Pelléas et Mélisande éclate comme une bombe. Il arrive du pays des bombes. Il est russe. Il est de Stravinski. C'est Le sacre du printemps [1924].

Cocteau reconnaît que Debussy et Stravinsky ont écrit des chefs-d'œuvre, mais il leur préfère néanmoins la musique d'Auric et de Poulenc :

Je ne vous ai pas dit que je brûle Wagner ni Beethoven ni aucun des compositeurs qui les inspirent ou qui en découlent. Je ne vous ai pas dit que Les biches ressemblaient à Cosi fan tutte ni Les fâcheux à Don Juan. J'ai dit grossièrement, et je le répète, que sauf Debussy et Stravinski dont les prodiges s'imposent, aucun musicien depuis Mozart n'avait su me satisfaire à l'orchestre autant que Georges Auric ou que Francis Poulenc ${ }^{15}$ [1924].

Lorsque Jean Cocteau rédige un compte rendu de La musique retrouvée 1902-1927 de Louis Laloy publiée chez Plon en 1928, il souligne le changement d'attitude de l'auteur, un ami et inconditionnel de Debussy, donc opposé aux Groupe des Six, qui s'est finalement rallié à ces derniers :

Laloy était en 1922 mon ennemi et l'ennemi des musiciens jeunes car l'amitié exclusive de Claude Debussy lui présentait nos antidotes volontaires, injustes, expéditifs (il fallait aller vite en besogne) comme des insultes à l'œuvre que nous chérissions et dont la place permettait qu'on l'attaquât sans lui porter atteinte.

Or, non seulement Laloy devint l'allié de nos moindres entreprises, mais encore il sut reconnaître Satie qui l'accablait de griefs mystérieux et ne désarma point. Cette haine maniaque ne l'empêche pas de saluer l'homme inénarrable dont l'œuvre plus légère que l'air s'élevait au-dessus du brouillard impressionniste, agaçait et ravissait Debussy [1929].

À la fin de la décennie 1920, Cocteau n'éprouve plus le besoin systématique de justifier ses prises de position exprimées dans Le Coq et l'Arlequin, mais celles-ci restent cependant présentées. En 1930, il va plus loin dans la contrition. Il se dit presque avoir été forcé de condamner l'impressionnisme afin de défendre la nouvelle musique française des Six :

15 Cocteau fait allusion aux deux œuvres créées au Théâtre de Monte-Carlo : Les biches de Francis Poulenc et Les fâcheux de Georges Auric. 
En 1916, il s'agissait de désenliser la musique. Je parle de la musique française. Nous dûmes même condamner Debussy (la révolution n'est pas toujours drôle), sous-estimer et surestimer des valeurs [1930].

Plus d'une fois, Cocteau évoque l'amitié qui liait Satie à Debussy, et les grogneries que le premier lançait au second. Un texte de 1959 en donne quelques détails croustillants :

Ces deux faunes barbus formaient une sorte de ménage amical avec ce qu'un ménage comporte d'amour hargneux et de disputes. Chaque dimanche, le vieil Erik déjeunait chez son vieux Claude et, à table, commençait une escrime de piques.

Satie qui n'aimait pas Ravel et disait de lui : " Il met la ponctuation en oubliant d'écrire dessous » allait jusqu'à louer les Valses nobles et sentimentales pour agacer Debussy qui ne les pouvait souffrir.

Ensuite, Satie, le chapeau melon sur l'œil, le binocle en bataille et son parapluie sur le bras, montait au 10 rue d'Anjou dans ma chambre, s'asseyait au pied de mon lit et, $\mathrm{du}$ coin de sa bouche sinueuse, sortaient des verdicts opposés à ceux de la mode et de l'avant-garde [1958].

Cocteau remarque avec justesse que Debussy a « ajouté de la sauce où il n'y en avait point, en orchestrant les Gymnopédies » [1959]. En même temps, ces deux versions sont - pour nous - des témoins ô combien parlant de styles très différents.

\section{AMENDEMENTS ET CONTRITIONS}

Les articles postérieurs à 1919 seront toujours des justifications, contritions et amendements divers par rapport aux aphorismes dénonciateurs du Coq et l'Arlequin. Cocteau ne se départira plus de cette attitude jusqu'à la fin des années 1930.

Dans la rubrique "Carte Blanche » de Paris-Midi qu'il débute en mars 1919, Cocteau entame une brève carrière de critique musical. Dans son premier article, il précise le but de la chronique qu'il anime, à savoir celui de tenir chaque semaine le lecteur au courant des "valeurs nouvelles » (1919). Debussy n'en fait pas partie : "L'impressionnisme a tiré son bouquet et alimente aujourd'hui les Beaux-Arts et le décor de théâtre ", ce qui signifie que le compositeur a lancé la dernière pièce d'un feu d'artifice. Par cette expression, Cocteau reconnaît la valeur même du compositeur, mais il le range parmi ceux qui appartiennent au passé.

Lors du premier concert des nouveaux jeunes sous le nom de "Groupe des Six » à la Galerie Montaigne, le 29 novembre 1920, Cocteau donne une allocution de présentation de ces jeunes musiciens. Il termine par la lecture d'un poème intitulé "À la mémoire de Claude Debussy », poème qui sera repris en 1922 dans le recueil de poésies Vocabulaire publié aux éditions La Sirène :

Les vagues, les feuilles, le vent

Et autres bêtes sans visage 
T'aiment, charmeur de paysages,

Et te savent toujours vivant.

Une Reine-Claude se tue

Sa blessure saigne de l'or

Marbre n'écrase pas ce mort

Dont un nuage est la statue (Cocteau 1999, p. 350).

On retiendra le terme de " charmeur » et sa variante « charme » qui seront ensuite abondamment utilisés par Cocteau lorsqu'il citera Debussy dans des textes ultérieurs. La présence de ce poème s'explique par le fait que Cocteau voulait faire paraître chez ce même éditeur un hommage à Debussy émanant du Groupe des $\mathrm{Six}^{16}$, mais ce projet fut abandonné, car La Revue musicale les avait devancés avec un supplément musical de dix compositions dédiées au compositeur défunt dans son numéro de décembre 1920. Cet hommage non réalisé prouve, si besoin est, que le Groupe des Six et son mentor gardaient une grande estime pour Debussy. À moins qu'il ne s'agisse d'une manouvre pour faire amende honorable.

Lorsque Cocteau défend les Six, il éprouve sans cesse le besoin de les opposer à l'impressionnisme, donc à Debussy, puisque c'est contre cette musique qu'ils se sont construits, mais il tempère ses propos vis-à-vis de Debussy par des nuances ambiguës, par exemple par le recours à l'oxymore, cette figure de rhétorique qui consiste à utiliser des mots opposés sémantiquement afin de provoquer un effet de surprise. Cocteau et chacun des Six ont prétendu que leur groupe n'était uni par aucune doctrine et que seule l'amitié les avait réunis, mais ce qui les unit, c'est de "rebâtir sur les décombres charmants [c'est nous qui soulignons] de l'impressionnisme » [1921], chacun dans une voie qui leur propre, sans caractéristiques communes.

Par ailleurs, il constate que la musique impressionniste a estompé et brouillé les formes anciennes, tels la fugue et le contrepoint par exemple que la jeune musique française remet à l'honneur avec des habits neufs. Dans l'histoire de la musique, ces formes évoluent comme des montagnes russes, elles vont et viennent au cours des temps :

Après la descente de Richard Wagner à Claude Debussy, qui donne un si profond et si agréable vertige, la pente est raide. Il faut remonter, l'accélération se calme, le cœur bat moins vite, nous distinguons mieux ce qui défile à droite et à gauche [1921].

En clair, le règne du flou, caractéristique de l'impressionnisme musical, cède la place à des formes plus structurées et à des lignes mélodiques plus claires.

Au cours de ses écrits, Cocteau sentira le besoin d'être de plus en plus nuancé vis-à-vis de Debussy et d'affirmer son admiration envers ce dernier. Lorsqu'en 1922, certains critiques musicaux tentent de semer la zizanie au sein du groupe des Six en isolant Arthur Honegger, Cocteau écrit une lettre ouverte à ses amis musiciens dans 
laquelle il met les points sur les « $\mathrm{i}$ ». La diaphore, figure de style qui réutilise un même mot dans deux significations différentes, permet au poète d'être incisif :

Celui de vous qu'on « organise » contre les autres participe encore quelquefois d'un ordre de choses à l'agonie. De cet ordre de choses il nous reste des idoles françaises. Le grand Debussy, par exemple. Car brûler une étape n'est pas brûler des idoles [1922].

Cocteau revient donc ici implicitement sur Le Coq et l'Arlequin et atténue ses propos à l'encontre de Debussy. Il va même jusqu'à prétendre que cette opposition d'alors à son égard est le fruit de l'interprétation des critiques eux-mêmes, ce qui relève d'une mauvaise foi évidente :

Une méthode naïve du monde musical que notre amitié dérange, consiste à nous montrer en lutte contre Debussy. Ce joli monde se trompe. Il lit mal. Il écoute mal. Mais vous, Monsieur, qui êtes attentif, devriez comprendre que vénérer, agir, sont deux choses [1922].

Cocteau s'étant exprimé, il est vrai, de manière à la fois si imagée et si brutale dans Le Coq et l'Arlequin, il devra constamment revenir sur ses aphorismes et tenter de les expliquer. Dans la querelle qui l'oppose à Paul Landormy par articles de presse interposés et qui repose sur la question de savoir si les Six forment un groupe homogène dont Cocteau serait le théoricien, ce dernier s'en défend, tandis que le critique en démontre la véracité. Cocteau en profite pour vouloir dissiper plusieurs malentendus :

J'aime et je respecte Claude Debussy. Un livre comme Le Coq et l'Arlequin n'aurait pas de sens s'il n'attaquait pas le debussysme puisque j'y montre les symptômes de sa contradiction. Livre trop bref pour se payer le luxe des demi-teintes. J'y parle gros, je m'exprime en majuscules ; si je blâme, je blâme des choses de premier ordre. Or, avec la poussière du tapis, le nettoyage par le vide risque d'arracher un peu de laine [1922].

De manière subtile, Cocteau distingue ici l'homme Debussy du compositeur Debussy. S'opposer à la musique impressionniste, ce n'est pas renier l'idole. Nous l'avons vu, Cocteau use des figures de style pour exprimer ses propos, mais il n'hésite pas non plus à user de mauvaise foi, allant même jusqu'à avancer des contre-vérités, celle, par exemple, d'avoir inventé la notion d'impressionnisme en musique $\left.{ }^{17}\right)$ :

17 Le terme d'« impressionnisme » est d'abord appliqué à la peinture de Claude Monet avec un sens péjoratif par le critique d'art Louis Leroy dans un article du Charivari paru en avril 1874 et qui s'inspirait d'une toile du maître intitulée Impression, soleil levant. Ce terme s'introduit également en littérature, puis en musique. En 1887, le rapport de l'Académie de France à Rome sur l'envoi du pensionnaire Debussy qualifie son morceau symphonique Le printemps d' "impressionnisme vague qui est un des plus dangereux ennemis de la vérité dans les œuvres d'art ». Cette date de 1887 est généralement retenue comme le premier emploi du terme impressionnisme appliqué à la musique, cette fois encore avec une connotation péjorative. 
Impossible d'empêcher que l'apparente une odeur d'époque et un instinct commun de réagir contre l'impressionnisme musical. Le terme " impressionniste " n'existait pas musicalement. Je le trouvai lorsqu'il fallut définir une période pour passer outre. Croyez-vous que passer outre veut dire brûler des idoles ? Même pas brûler des étapes. Debussy reste notre poète, il ne faudrait pas l'écraser sous un marbre car son buste est un nuage merveilleux. N'oublions pas le Général Lavine et Ministrels où il invente le rythme caricatural [1922].

Ce que l'on retient ici, ce sont les nuances positives et favorables que Cocteau apporte aux thèses défendues dans Le Coq et l'Arlequin: le nuage, le flou sont donc à présent décrits comme " merveilleux ». Il reconnaît aussi un élément de modernité dans la musique de Debussy, celui de ce rythme inhabituel du rag-time dans les deux pièces citées ${ }^{18}$.

Lors de l'inauguration des deux monuments érigés à la mémoire de Debussy en juin 1932, respectivement dans le $\mathrm{XVI}^{\mathrm{e}}$ arrondissement et à Saint-Germain-en-Laye, et qui réunit le tout Paris politique et artistique, Cocteau reste curieusement muet, absent de Paris à cette époque.

\section{RAVEL OUBLIÉ DE COCTEAU PARMI LES IMPRESSIONNISTES ?}

Jusqu'ici, les compositeurs appartenant à l'impressionnisme musical se limitaient, sous les doigts de Cocteau, au seul Debussy, et encore bien souvent par de simples allusions. Ce n'est qu'en 1937, lors de la disparition de Maurice Ravel que Cocteau le cite dans un texte d'hommage.

Ce dernier est cependant ponctué, car selon la théorie coctalienne des chefs-d'œuvre qui engendrent des suiveurs, Ravel apparaît comme " le chef des petits-maîtres de l'impressionnisme ». Ses trouvailles le rapprochent cependant des Six :

Ravel a, pour ainsi dire, raffiné l'art des grands maîtres impressionnistes de la musique, de même que Vuillard et Bonnard, auxquels il s'apparente, ont compliqué, simplifié, affermi le style des grands impressionnistes dont Monet demeure l'emblème.

Ravel est, en quelque sorte, le chef des petits-maîtres de l'impressionnisme et, non pas par son manque de sauce, mais par les mélanges savants et les épices de sa sauce, il se rapproche déjà des jeunes qui allaient suivre, sous la

\footnotetext{
Pourtant, le critique musical belge Maurice Kufferath utilise déjà cette expression l'année précédente pour qualifier les mélodies de Franz Servais, mais cette fois dans une perception positive : "C'est son rêve qu'il [Servais] nous conte. Mais écoutez-bien, car les rêves ne se disent pas en la langue vulgaire de tous les jours. Cela n'aura ni logique, ni bon sens, et cependant cela charmera. On se demande s'il s'agit ici d'une composition savamment combinée ou d'une improvisation désordonnée. Ce n'est ni tout à fait l'un, ni tout à fait l'autre. [...] C'est de l'impressionnisme musical » (M. K. 1886, p. 343-344).

18 Parmi les pièces aux rythmes de jazz composées par Debussy, on peut également ajouter « Golliwog's Cake Walk", la sixième des Chridren's Corner.
} 
bannière d'un homme qui n'était plus jeune, qui était sans âge : Erik Satie, et qui inventa de rompre avec l'impressionnisme musical en se mettant, déjà vieux, à l'école de Vincent d'Indy, du contrepoint, de la fugue, bref de la Schola Cantorum [1938].

Et Cocteau de se rappeler les soirées passées chez les Cipa Godebski où ils écoutaient Ricardo Viñes jouer à la fois du Satie, du Debussy et du Ravel (Haine 2006). Cocteau n'est donc pas spécialement admiratif puisqu'il classe le compositeur de Ma mère l'Oye dans la catégorie de ceux qui gravitent autour des maîtres. Par contraste, Debussy en ressort alors grandit.

Comme toujours chez Cocteau, les remords viennent ensuite. Dans l'ouvrage d'hommages collectif intitulé De la musique encore et toujours!, publié en 1946, 1'acte de repentir se mue en une véritable admiration dès le titre : "Maurice Ravel salué par Jean Cocteau ». La jeunesse, une fois de plus, est appelée à la rescousse, pour justifier les propos d'antan, mais ceux-ci se transforment finalement par une admiration commune pour Debussy, Ravel, Satie et Stravinsky :

J'ai été très injuste pour Ravel et quelquefois même de cette férocité propre à la jeunesse. Il le fallait. Les gens qui nous relisent oublient trop souvent nos guerres des lettres et nos politiques.

J'aimais Ravel. Mais il importait d'en finir au plus vite avec l'impressionnisme musical. Ravel venait de tirer le bouquet de ce feu d'artifice. Il ne pouvait plus y avoir que fin de courbe. [...]

Maintenant, l'âge me donne le recul et le large. J'écoute Ravel dans le calme et je l'admire. Je suis libre de l'admirer et de le mettre à sa place. Elle est considérable. Aux côtés de Vuillard et de Bonnard, pour qui nous dûmes aussi être injustes, il miroite gravement de mille feux déformés par l'eau.

Salut Debussy ! Salut Ravel ! Salut Satie ! Salut Stravinski ! Salut hautes cimes rejointes par des vallées d'ombre [1946].

Le 4 novembre 1953 se tient un concert commémoratif du Groupe des Six au Théâtre des Champs-Élysées. Cocteau y fait une allocution dans laquelle Ravel est cité comme faisant partie du groupe amical que fréquentaient les jeunes musiciens. Ravel serait-il cette fois réhabilité aux yeux du poète, lui qui n'a jamais mentionné son nom, dans aucun de ses articles, du vivant du compositeur? :

Entre Debussy, Ravel et les jeunes qui se recommandent de Schoenberg régna ce groupe libre où l'amitié tenait lieu d'esthétisme. Une famille plutôt qu'une école, une marche vers cet épanouissement que consacre la séance solennelle de 1953 [1953].

\section{COMMÉMORATIONS ET SOUVENIRS APRÈS 1940}

Après 1940, nous entrons dans l'ère des commémorations et des souvenirs. Les éloges quasi inconditionnels remplacent à présent les attaques et justifications. 
C'est le cas en 1942 lors d'une exposition sur Debussy organisée par l'OpéraComique. Le catalogue renferme un petit texte délicieux de deux paragraphes:

Debussy existait avant Debussy. C'étaient une architecture qui bouge à l'envers dans l'eau, des nuages qui se construisent et qui s'écroulent, des branches qui s'endorment, la pluie sur les feuilles, des prunes qui tombent, qui se tuent et qui saignent de l'or. Mais tout cela murmurait, bégayait, n'avait pas trouvé une voix humaine pour se dire. Mille vagues merveilles de la nature ont enfin découvert leur traducteur.

Si j'ai, jadis, mis la jeunesse en garde contre Claude Debussy, c'est qu'un génie pareil ouvre une porte et la referme. On se perdrait à vouloir le suivre. Il est un monde perfide et prodigieux [1942].

Cocteau avait sans doute envisagé un texte de souvenirs à la place de celui cité ci-dessus. Un manuscrit inédit, intitulé "Souvenirs ", date vraisemblablement de la même époque. Retenons quelques expressions qui contrastent avec les aphorismes sévères de 1919 : " œuvre exquise », " amour de Debussy », " le propre du génie est d'être intemporel ».

La virulence des propos du Coq et de l'Arlequin sont à expliquer, écrit Cocteau, par les emportements excessifs de la jeunesse « qui ne supporte pas qu'on la domine et qui se croit malade lorsqu'elle succombe à l'amour ». N'est pas ici une véritable déclaration d'amour justement? Voici le texte intégral de cet inédit :

Les circonstances m'ont toujours obligé de prendre en face de cette ouvre exquise une attitude hostile. C'est le propre de la jeunesse que de mettre l'esprit de contradiction en œuvre et que de rejeter comme détestable ce qui dérange son message.

Trop de sauce, de brumes et de voiles nous poussaient vers la ligne mélodique et vers une simplicité apparente. Claude Debussy en souffrait et jusqu'à sa mort il crut que nous souhaitions le voir disparaître avec son faune et ses nymphes.

En vérité nous étions envahis par son règne et nous défendions nos prérogatives avec cette monstrueuse injustice de la jeunesse qui ne supporte pas qu'on la domine et qui se croit malade lorsqu'elle succombe à l'amour.

C'est l'amour de Claude Debussy, c'est son élément mêlé à notre organisme qui nous paralysait et nous obligeait à la révolte.

La sauvage croissance du Sacre nous dictait une attitude violente et, même, ensuite, nous nous tournâmes contre ce Sacre qui avait été notre étendard.

L'âge calme toutes ces tempêtes et, maintenant, nous sommes aptes à nous reposer sur la mousse, à écouter la flûte du faune. Notre maison est assez riche pour recevoir noblement un hôte royal.

Erik Satie, s'il vivait, saluerait avec nous son vieux camarade et rirait des disputes qui les excitaient l'un contre l'autre à la table du dimanche. 
Le propre du génie est d'être intemporel. Le progrès n'a pas de prise sur son phénomène. Claude Debussy ajoute un élément à ceux qui nous permettent de vivre en ce monde [?1942].

Le numéro spécial de juin 1952 de La Revue musicale consacré à " Erik Satie. Son temps et ses amis ", permet à Cocteau de reconnaître les deux maîtres musicaux $\mathrm{du} \mathrm{Xx}^{\mathrm{e}}$ siècle, tout en mettant Satie au pinacle :

Si l'on m'interrogeait sur notre époque, je répondrais sans doute que les plus grands sont Debussy et Stravinski. Et j'ajouterais : "Mais Satie est l'unique » [1952].

Signalons ici l'emprunt de Cocteau au bon mot de la princesse de Belgiojoso à l'issue du combat pianistique entre Franz Liszt et Sigismond Thalberg qui s'était tenu dans ses salons en 1837 : "Thalberg est le premier pianiste au monde, mais Liszt est l'unique ».

La glorification de Debussy atteint son paroxysme dans un très court texte destiné à accompagner la sortie d'un disque du Prélude à l'après-midi d'un faune en 1957 :

Claude Debussy ou « le dieu Pan ». Il a, sur sa flûte, donné une voix au vent, aux vagues, aux nuages.

Il a fait murmurer les monstres marins et sous l'arbre aux fées, il a fait entendre des voix de France que seule Jeanne d'Arc avait entendues.

Nous l'aimions parce qu'il est amour et mystère [1956].

En 1962, Cocteau revient encore sur ces attaques de jeunesse dans un numéro spécial du Journal musical de France consacré à Debussy et qui seront repris un an jour pour jour, lors du décès même de Cocteau :

Lorsque nous attaquions jadis Debussy et la musique impressionniste, c'était non seulement à cause de cette insolence de l'extrême jeunesse, mais encore parce que la jeunesse croit se faire une infidélité en se laissant envahir par l'amour d'une œuvre.

Tout cela est fort loin et l'âge nous a rendus dignes d'ouvrir notre âme à l'hôte royal.

Debussy possède cet étrange privilège de se mélanger aux forces et aux grâces mystérieuses de la nature. On le salue comme le vent, les arbres, les nuages, les fleuves, la mer [1962]. 
PELLÉAS ET MÉLISANDE ${ }^{19}$

Mis à part l'aphorisme dans lequel Cocteau exprime l'affiliation wagnérienne de Debussy (voir ci-dessus « la musique qui s'écoute la figure entre les mains » comme celle de Wagner), le poète a rarement cité directement l'œuvre majeure de Debussy avant les années 1940.

Dans un texte datant de 1945, il consacre deux paragraphes à Pelléas qu'il qualifie de " chef-d'œuvre ", mais tempère immédiatement son appréciation en lui donnant le qualificatif de «poétique ». Parler de « chef-d'œuvre poétique », c'est goûter le texte et non la musique, d'autant que Cocteau explicite clairement cette notion, lui qui reste généralement assez vague dans l'emploi de ses allégories ou métaphores :

Le Pelléas de Debussy est un chef-d'œuvre poétique. Le texte de Maeterlinck un chef-d'œuvre de poésie. Ce que l'opéra dégage de poésie vient beaucoup plus du dramaturge que du musicien. Mais les gens ne s'en rendent pas compte. Ils croient que le poète est inférieur à la musique et même, sans la musique, un peu ridicule et démodé.

Alors que c'est justement ce qui déplaît encore en Maeterlinck qui le sauve et que l'accès trop rapide et trop unanime des élites à la musique de Debussy, nous prouve qu'elle est de la prose poétique sur la poésie en prose de Maeterlinck [1945].

En 1952, le cinquantenaire de la création de Pelléas est salué par maintes représentations de l'œuvre sur les scènes lyriques du monde entier. La reprise à l'OpéraComique de Paris est conforme à la création de 1902 : la mise en scène d'Albert Carré et Albert Vizentini est reconstituée par Louis Musy, les décors de Lucien Jusseaume et Eugène Ronsin sont restitués par Raymond Deshays, tandis que les costumes sont repris de Charles Bianchini (Charlton et Wild 2005, p. 359).

Cocteau ne peut rester absent de ces manifestations et salue la représentation dans Les Lettres françaises. Le titre de son article repris en dernière phrase ne peut laisser indifférent : "Pelléas et Mélisande, phénomène unique dans l'histoire de la poésie » est destiné à frapper les esprits et à clamer bien haut l'admiration portée au compositeur. Pourtant, le contenu de l'article est assez équivoque : la poésie et la musique semblent être mises sur le même pied ; elles sont toutes deux qualifiées de chefs-d'œuvre, mais l'une a mangé l'autre. Debussy et sa musique ont phagocyté Maeterlinck et sa poésie, telle une " mante-religieuse » qui mange l'autre par amour.

On apprend aussi que Cocteau a été sollicité pour s'occuper de la mise en scène, des costumes et des décors, mais que faute de temps, il n'a pu accepter :

19 Rappelons que Pelléas et Mélisande, drame lyrique en cinq actes de Maurice Maeterlinck sur une musique de Claude Debussy, est créé le 30 avril 1902 au Théâtre national de l'Opéra-Comique à Paris sous la direction musicale d'André Messager, dans une mise en scène d'Albert Carré et Albert Vizentini, avec des décors de Lucien Jusseaume et d'Eugène Ronsin et avec des costumes de Charles Bianchini. 
Il est rare que deux chefs-d'œuvre se joignent et s'épousent. Si la chose arrive, il n'est pas rare qu'un chef-d'œuvre mange l'autre comme une mante religieuse en amour. Dans le phénomène Pelléas, pas le moindre pléonasme. Impossible, en outre, de dire qu'une âme musicale s'est introduite dans un corps. C'est un mélange mystérieux de corps et d'âmes. Il n'en reste pas moins vrai que si Gounod n'a pas mangé Goethe ${ }^{20}$, Debussy a mangé Maeterlinck et que pour nombre de personnes l'admirable pièce de théâtre n'est plus qu'un livret d'opéra. C'est même sous cette forme qu'on l'édite.

Lorsque l'Opéra-Comique m'a demandé la nouvelle mise en scène, les costumes et les décors, je n'étais, hélas, pas libre. J'avais proposé de reprendre les vieux décors pour qu'ils devinssent une sorte de Vuillard, ce qui arrive avec le recul.

Les décors de Valentine Hugo ${ }^{21}$ supprimaient volontairement un troisième risque de pléonasme. Il est dommage qu'on les écarte. On les reprendra.

Il faut saluer en Pelléas et Mélisande mieux qu'un chef-d'œuvre : un phénomène unique dans l'histoire de la poésie [1952].

$\mathrm{Si}$, en 1952, Cocteau n'a point eu le temps de répondre favorablement aux sollicitations de l'Opéra-Comique, il acceptera dix ans plus tard de s'impliquer autrement que par l'écrit. Lors des commémorations du centenaire de la naissance de Debussy, plusieurs productions de Pelléas et Mélisande sont à nouveau montées un peu partout en Europe et aux États-Unis ${ }^{22}$.

Cocteau conçoit les décors et les costumes pour les représentations au Théâtre municipal de Metz, dont la première a lieu le 22 septembre 1962. Henri Doublier se charge de la mise en scène, tandis que Jacques Pernoo assure la direction musicale. Ce spectacle fait l'objet d'un entretien radiophonique avec Henri Haton pour Radio-Lausanne déjà évoqué ci-dessus. Cocteau reprend le thème de deux chefsd'œuvre dont l'un à manger l'autre. Toutefois, de nouvelles idées émergent, et il justifie aussi ses décors :

[...] La musique impressionniste est féminine, mais... mâle le texte de Maeterlinck. Il résulte de ce mariage que, comme chez les Mantes Religieuses, (sourire) l'épouse dévore l'époux pendant l'acte d'amour.

Le chef-d'œuvre de Claude Debussy a dévoréle chef-d'œuvre de Maeterlinck, à tel point qu'il arrive à la comtesse Maeterlinck de se plaindre parce que le nom du dramaturge ne figure même pas sur les affiches.

20 Allusion au Faust de Gounod.

21 Cocteau parle ici des décors dessinés par Valentine Hugo pour la reprise de l'œuvre en 1947 à l'OpéraComique qui avaient provoqué la fureur du public et des interprètes, car ils reflétaient un symbolisme dont les caractéristiques n'avaient aucune relation avec le sujet de l'œuvre (Langham Smith et Nichols 1988, p. 159).

22 En dehors de Paris, la production la plus originale a lieu à Lyon par le metteur en scène Louis Erlo, disciple de Wieland Wagner. 
Je tiens de Claude Debussy, qu'il ne voulait pas composer un opéra mais souligner, par une ligne musicale, le relief des paroles.

C'est pourquoi si les chanteurs ne sont pas des acteurs ou sont des acteurs médiocres et articulent mal, l'œuvre devient longue, et ennuyeuse.

[...] Pour les décors de Pelléas, [...] j'ai enfin risqué ma chance pour trois raisons.

La première c'est que j'étais trop jeune à l'époque de la création d'une œuvre que je devinais à travers des programmes rapportés par ma famille. Je trouvais le programme de Pelléas sur mon lit au réveil et je passais d'un rêve dans un autre.

C'est ce qui me reste en mémoire des vieux décors de Jusseaume que j'ai voulu fixer par des esquisses rapides sur des surfaces transparentes que la lumière traverse.

La seconde raison me vient de Claude Debussy. Un soir, la veille - ou l'avantveille (sourire) - du Martyre de Saint-Sébastien, comme nous parlions au bar de l'Opéra du casse-cou des décors de Pelléas, il me dit : (imitation de voix) «Il faudrait un peintre japonais pour les réussir » (il avait composé sa musique à l'époque du japonisme, du japonisme mallarméen).

La troisième raison fut le style des vignettes de Manet qui illustrent la plaquette luxueuse de L'après-midi d'un faune. Plaquette dont Mallarmé désirait qu'elle ressemblât « à un sac de bonbons, mais de rêve " ${ }^{23}$.

En outre, je... je charge le costume médiéval de mettre formes et couleurs dans cette brume matinale et crépusculaire.

Il est possible, après un si grand nombre de tentatives que la mienne... déçoive à la fois les amateurs de réalisme et les amateurs de songe. Mais l'œuvre est écrite à cheval sur ces contrastes et il fallait obéir à l'élégance suprême de ce déséquilibre (Héron 2010, p. 78).

Le texte d'introduction au programme, rédigé par Cocteau, reprend des idées exprimées précédemment, mais avance aussi de nouveaux propos :

Lorsque le chef-d'œuvre de Maeterlinck fut épousé par un autre chefd'œuvre ${ }^{24}$, il naquit de cet accouplement étrange, un scandale ${ }^{25}$ ).

23 Le texte de L'après-midi d'un faune a paru chez A. Derenne en 1876 avec frontispice, fleurons et cul-de-lampe par Édouard Manet. Dans la note bibliographique de ses Poésies (1898), Mallarmé note : "L'après-midi d'un faune parut à part, intérieurement décoré par Manet, une des premières plaquettes coûteuses et sac à bonbons mais de rêve et un peu orientaux avec son "feutre du japon, titré d'or et noué de cordons rose-de-Chine et noirs", ainsi que s'exprime l'affiche : puis M. Dujardin fit, de ces vers introuvables autre part que dans sa photogravure, une édition populaire, épuisée»» (consultation en ligne voir bibliographie).

24 Cocteau fait allusion ici au Sacre du printemps de Stravinsky.

25 Scandale que Cocteau explique, dans l'entretien avec Jaton, de la façon suivante : «En réalité, en baignant le texte de Maeterlinck dans une sorte de vague musical, Debussy contredisait la théorie des airs 
J'étais alors trop jeune pour suivre ma famille au théâtre. Elle en revenait pendant mon sommeil et laissait dans ma chambre le programme ou un superbe magazine en couleurs : Le Théâtre.

Si j'ai toujours refusé de faire les décors et les costumes de Pelléas à l'OpéraComique, au Metropolitan Opera de New York, à celui de Londres, à la Scala de Milan, malgré la prière affectueuse de Madame Maeterlinck, c'est par peur de perdre l'équilibre entre le rêve et un pur réalisme qu'une longue méprise fait encore confondre avec l'irréalité. Lorsque je m'efforce à Metz de vaincre mes scrupules, c'est qu'il m'a paru curieux de rendre hommage aux décors d'origine de Jusseaume en dessinant vite et presque les yeux fermés, le souvenir d'enfance que j'en garde. Cette méthode permet en outre de résoudre le lourd problème du machinisme et, sur des tulles, d'accentuer le relief du style médiéval des costumes auquel je songeais sur les neiges d'Engadine en regardant skieurs et skieuses pareils aux archers et aux belles dames de Dürer, Cranach ou Holbein.

Je n'ignore pas le rôle que jouent les cheveux de Mélisande [Allusion à la scène 2 de l'acte IV, où Golaud traîne Mélisande par les cheveux], mais les lourdes perruques m'effraient et je préfère la coiffer avec ces charmantes cagoules, sur lesquelles, au Moyen Âge, nos reines portaient leurs couronnes.

Puisse la profonde et douce lumière de Maeterlinck traverser cette ruche et m'éviter d'alourdir le vol nuptial de Mélisande semblable à celui des abeilles ${ }^{26}$ [1962].

La participation de Cocteau à la production scénique d'une œuvre de Debussy n'est-elle pas un acte de contrition qui a valeur de réparation, voire de rédemption?

\section{CONCLUSION}

Avec la publication du Coq et l'Arlequin, Cocteau veut promouvoir Satie et les jeunes musiciens qui l'entourent. Pour tenter de les imposer, il faut s'opposer au chef de file de l'époque, à savoir Debussy. Les aphorismes qui attaquent celui-ci sont assez virulents; aussi le public retiendra-t-il que Cocteau n'apprécie guère la musique de Debussy. Par la suite, il se justifiera en se cachant derrière la fougue d'une jeunesse désireuse de renier les valeurs reçues.

Que ce soit dans les textes traitant d'Erik Satie, d'Igor Stravinsky ou des membres du Groupe des Six, le nom de Debussy se glisse à chaque fois sous la plume du poète tant pour louer le compositeur français que pour justifier les prises de positions de cet écrit de jeunesse.

qu'on retient du bel canto, et alors les gens n'avaient pas de points de repère et se moquaient beaucoup surtout, des situations [scéniques] assez bizarres [...]» (Héron 2010, p. 79).

26 Selon Langham Smith et Nichols (1988, 162), cette production de Metz est reprise à 1'OpéraComique de Paris l'année suivante. 
À maintes reprises, Cocteau s'efforce de démontrer qu'il admire le compositeur et son chef-d'œuvre, Pelléas et Mélisande, dont il conçoit même les décors et les costumes lors d'une production au Théâtre municipal de Metz en 1962.

Remarquerons enfin que si Cocteau a, dans ses premiers écrits sur la musique, refusé de reconnaître à la musique de Debussy les caractéristiques d'une musique française dépourvue des influences germaniques ou russes, Debussy a pris place au panthéon des musiciens français, au point d'être surnommé « Claude de France ». N'est-ce pas là une belle revanche?

\section{BIBLIOGRAPHIE}

Charlton, David, et Nicole Wild (2005), Théâtre de l'Opéra-Comique [de] Paris. Répertoire 1762-1972, Sprimont, Mardaga.

Cocteau, Jean (1918), Le Coq et l'Arlequin. Notes autour de la musique, Paris, La Sirène.

Cocteau, Jean (1999), Euvres poétiques complètes, éd. Michel Décaudin, Paris, Gallimard.

Gullentops, David, et Malou Haine (dir.) (en préparation), Écrits de Jean Cocteau sur la musique et la danse, Paris, Vrin.

Haine, Malou (2006), "Cipa Godebski et les Apaches ", Revue belge de Musicologie, vol. 60, p. 221266.

Haine, Malou (à paraître), « Parade de Jean Cocteau. Un exemple de transpositions intersémiotiques », Timothé Picard (dir.), Les contributions d'écrivains dans la presse musicale (XXX-XXI siècles).

Héron, Pierre-Marie (2010), " [Entretien radiophonique de Jean Cocteau] Avec Henri Jaton (1962) ", Jean Cocteau et la radio, Cahiers Jean Cocteau, $n^{\circ}$ 8, p. 77-85.

Langham Smith, Richard, et Roger Nichols (1988), Claude Debussy. Pelléas et Mélisande, Cambridge, Cambridge University Press.

Lesure, François (2003), Claude Debussy. Biographie critique suivie du Catalogue de l'aeuvre, Paris, Fayard.

M. K. [Maurice Kufferath] (1886), " Le concert de M. Franz Servais », Le Guide musical, vol. 48 (2 décembre), p. 343-344.

Mallarmé, Stéphane (1876), L'après-midi d'un faune, Paris, Alphonse Derenne, https://fr.wikisource. org/wiki/L'Après-Midi d'un faune/Édition 1876, consulté le 12 janvier 2012.

Mallarmé, Stéphane (1898), Poésies, http://fr.wikisource.org/wiki/Poésies (Stéphane Mallarmé) Bibliographie, consulté le 12 janvier 2012.

Orledge, Robert ([1990]1992), Satie the Composer, 2e édition, Cambridge, Cambridge University Press. 